\title{
Enhancement of Photocatalytic Water Splitting Rate via Rayleigh Convection
}

\author{
Seiichi Deguchi'1, Ben Kariya², Norifumi Isu'3, Shoji Shimasaki', Haruna Banno', \\ Saeko Miwa1, Keisuke Sawada', Junki Tsuge1, Shoichiro Imaizumi', Hidenori Kato', \\ Kyota Tokutake ${ }^{1}$ \\ ${ }^{1}$ Department of Energy Engineering and Science, Nagoya University, Nagoya, Japan \\ ${ }^{2}$ School of Chemical Engineering, Purdue University, West Lafayette, USA \\ ${ }^{3}$ Kitchen and Bathroom Technology Research Institute, LIXIL Corporation, Tokoname, Japan \\ Email: deguchi@nuce.nagoya-u.ac.jp
}

Received 30 October 2013; revised 28 January 2014; accepted 10 March 2014

Copyright (C) 2014 by authors and Scientific Research Publishing Inc.

This work is licensed under the Creative Commons Attribution International License (CC BY).

http://creativecommons.org/licenses/by/4.0/

(c) (i) Open Access

\begin{abstract}
In order to enhance photocatalytic water splitting rates with $\mathrm{Pt} / \mathrm{TiO}_{2}$ powder, sufficient agitation of the biphasic medium is required to switch surficial reactions to volumetric reactions. Additionally, agitation is conducive to higher diffusion rates of the generated hydrogen and co-produced oxygen, hindering their ability to re-couple to water on $\mathrm{Pt}$ loaded to $\mathrm{TiO}_{2}$ powder. In order to create agitation without consuming any electricity, a novel technique utilizing Rayleigh convection was applied, and its ability to enhance photocatalytic water splitting rates was evaluated. Higher Rayleigh convective flow rates resulted in higher photocatalytic water splitting rates. Utilization of Rayleigh convection approximately doubled the photocatalytic water splitting rates, despite relatively low convective flow velocities (obtained through simple thermo-hydrodynamic simulations). The rate enhancement achieved through Rayleigh convection is a result of its ability to disperse the ultrafine $\mathrm{Pt} / \mathrm{TiO}_{2}$ particles throughout the whole medium, leading to volumetric reactions.
\end{abstract}

\section{Keywords}

Photocatalytic Water Splitting, Pt/TiO 2 Powder, Rate Enhancement, Volumetric Reaction, Thermal Treatment, Rayleigh Convection, Thermo-Hydrodynamic Simulation

\section{Introduction}

Photocatalysts play important roles in a variety of applications, namely in water decontaminations, air pollutants degradations and water splitting [1]-[9]. Concerning photocatalytic water splitting, $\mathrm{Pt} / \mathrm{TiO}_{2}$ powder is the most 
promising photocatalyst, as the band structures of $\mathrm{TiO}_{2}$ are particularly well suited for water splitting and Pt loaded induces the Schottky effect.

$\mathrm{Pt} / \mathrm{TiO}_{2}$ powder precipitates easily in standing aqueous solutions of some oxidizing sacrificial agents (OSAs), resulting to decrease in interfacial area between the $\mathrm{Pt} / \mathrm{TiO}_{2}$ powder and the solution, and slower hydrogen producing rates due to surficial reactions as well. In the former works, the efficient OSA of castor oil with an undesirable characteristic of water-insoluble and absorption/reflection of ultraviolet rays due to the solution before reaching the precipitated $\mathrm{Pt} / \mathrm{TiO}_{2}$ powder had been found [1] [2] [7] [9]. In order to overcome these obstructive matters described-above, agitation of the mixture of $\mathrm{Pt} / \mathrm{TiO}_{2}$ powder and OSA solution must be a great help to disperse $\mathrm{Pt} / \mathrm{TiO}_{2}$ powder through the whole solution, resulting to volumetric reactions and then higher $\mathrm{H}_{2}$ producing rates expected. Here, water electrolysis is quite efficient (above $90 \%$ in a converting efficiency), and then it played important roles in the WE-NET [10] (World Energy Network) project using electric power generated from renewable energy sources such as hydropower and wind power, especially in Canada and northern Europe, respectively. Because of such a high efficiency of water electrolysis, agitation of the mixture of $\mathrm{Pt} / \mathrm{TiO}_{2}$ and OSA solution must be realized without consuming any electricity.

In this study, Rayleigh convective flows induced by waste heat in low temperatures (less than $373 \mathrm{~K}$ ) as one of the agitating candidates with no electric power consumptions had been applied to water splitting by $\mathrm{Pt} / \mathrm{TiO}_{2}$ powder. Experiments together with simple theoretical calculations were carried out to verify the effectiveness of Rayleigh convection in enhancing photocatalytic water splitting rates. Though the Rayleigh convective flow rates induced by partial heating operations were found rather low, enhanced photocatalytic water splitting rates were achieved. Finally, promising future strategies for higher agitating intensities under lower temperatures, which might result to further enhancement of photocatalytic water splitting rates, are also discussed.

\section{Experimental}

All materials and equipments utilized in this study aside from the main experimental apparatus are summarized in Table 1. Regarding key materials, 50 vol\% methanol aqueous solution and $0.10 \mathrm{wt} \% \mathrm{Pt}$ loaded $\mathrm{TiO}_{2}$ powders (P25 and P90 from Nippon Aerosil Co., Ltd.), that were sufficiently grinded (ANM1000, Nitto Kagaku Co., Ltd.), were used throughout the experiments.

Figure 1 shows a schematic of the experimental apparatus. It was mainly made of transparent acrylic resin in the shape of a square horizontal cross section with $270 \mathrm{~mm}$ in a side and a height of $50 \mathrm{~mm}$. It also had two taps for replacing air inside initially with argon, the top cover made of solid quartz glass and the aluminum plate bottom in order to obtain a higher rate of heat transfer and high reflectance toward light in all wavelength ranges. Beneath the aluminum plate, preheated water was circulated excluding parts where adiabatic Styrofoam blocks were attached to the plate. Each Styrofoam block had $30 \mathrm{~mm}$ wide, $90 \mathrm{~mm}$ long and $10 \mathrm{~mm}$ in height with 30 $\mathrm{mm}$ gaps each other to form temperature gradients at the upper surface of the plate, inducing Rayleigh convection in the test medium. It should be emphasized that any equipment consuming electricity must be excluded in

Table 1. Materials and equipment utilized.

\begin{tabular}{|c|c|c|c|}
\hline & Material or equipment & Composition or code & Manufacturing company \\
\hline \multirow{4}{*}{$\begin{array}{l}\text { Pt loading } \\
\text { to } \mathrm{TiO}_{2}\end{array}$} & Pt source & $\mathrm{H}_{2} \mathrm{PtCl}_{6}$ & Kanto Kagaku \\
\hline & Air pump & OX-30 & Tetra Japan \\
\hline & Auto grinder & ANM1000 & Nitto Kagaku \\
\hline & Muffle furnace & MMF-2 & AS ONE \\
\hline \multirow{4}{*}{$\begin{array}{c}\text { Above } \\
\text { and } \\
\text { below }\end{array}$} & Photocatalyst & P25 and P90 & Nippon Aerosil \\
\hline & Reducing agent or OSA & $\mathrm{CH}_{3} \mathrm{OH}$ & Wako Pure Chemical \\
\hline & Black light & EFD15BLB-T & Toshiba \\
\hline & Magnetic stirrer & HS-360 & AS ONE \\
\hline \multirow{5}{*}{$\begin{array}{c}\text { Photocatalytic } \\
\text { water } \\
\text { splitting }\end{array}$} & Initial gas phase control & $\mathrm{Ar}$ & Alpha System \\
\hline & Fluorescent lamp & FL-40SS EX-N/37-H & TOSHIBA \\
\hline & Water bath & TR1 & IUCHI \\
\hline & Water pump & MD-15R & IWAKI \\
\hline & Gas chromatography & TCD/GC-8A & SHIMADZU \\
\hline
\end{tabular}




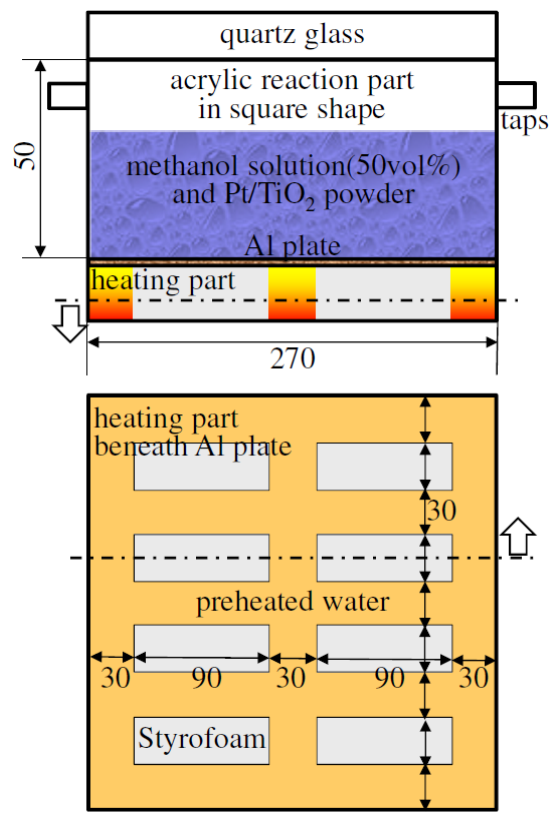

Figure 1. Schematic drawing of experimental apparatus.

photocatalytic water splitting reactions to be practical utilization. Then, both waste heating media with pre-existing momentums and visible/infrared rays are considered promising candidates for inducing Rayleigh convection. The heating part could be replaced to a magnetic stirring system for comparative experiments.

Methanol aqueous solution at $298 \mathrm{~K}$ was initially poured into the reactor along with the $\mathrm{Pt} / \mathrm{TiO}_{2}$ powder. After beginning the circulation of the preheated water to the reactor bottom, light irradiation was initiated to start the experiment. During the experiment, concentration of $\mathrm{H}_{2}$ in the gas phase of the reactor was intermittently measured.

\section{Simulation Procedures}

Figure 2 shows the smallest possible cross-sectional area for two-dimensional theoretical thermo-hydrodynamic simulations in the case of $20 \mathrm{~mm}$ depth of solution, assuming symmetrical flow and ignoring wall effects. This calculated area was equally divided into a grid with 10 rows and 20 columns of rectangles $2 \mathrm{~mm}$ high and $3 \mathrm{~mm}$ wide each. Detailed descriptions of governing equations and calculations were omitted from this paper. In short, equations of continuity, Navier-Stokes and unsteady state heat transfer were solved by the HSMAC (Highly Simplified Marker-and-Cell) method with Staggered grids [11]-[15].

\section{Results and Discussion}

\subsection{Effect of Solution Depth and Agitation}

Figure 3 shows photocatalytic $\mathrm{H}_{2}$ production with various solution depths of 10,20 and $30 \mathrm{~mm}$ without agitation as well as with agitation by magnetic stirrers at a depth of $30 \mathrm{~mm}$. Without agitation, deeper solutions show slower $\mathrm{H}_{2}$ producing rates and longer dead times before any $\mathrm{H}_{2}$ production. These results could be attributed to the undesirable phenomena described in the introduction (i.e. light absorption/reflection by the solution and surficial reactions on the precipitated $\mathrm{Pt} / \mathrm{TiO}_{2}$ powder). Despite $30 \mathrm{~mm}$ of the deepest solution among tested, benefits of agitating the solution were confirmed from the shortest dead time and the highest $\mathrm{H}_{2}$ production rate, which was approximately double rate seen in the case of the $10 \mathrm{~mm}$ deep solution without agitation.

The effectiveness of agitation on enhancing photocatalytic water splitting rates is quite conclusive. Thereby, justifying interests in Rayleigh convection as a promising method of enhancing photocatalytic water splitting rates well out because it does not consume any power. 


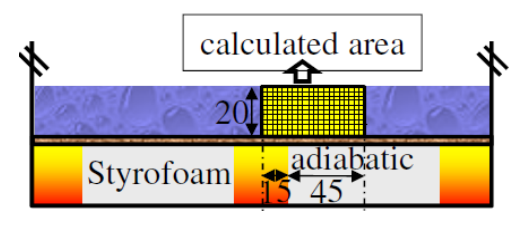

Figure 2. Physical configuration for theoretical calculations.

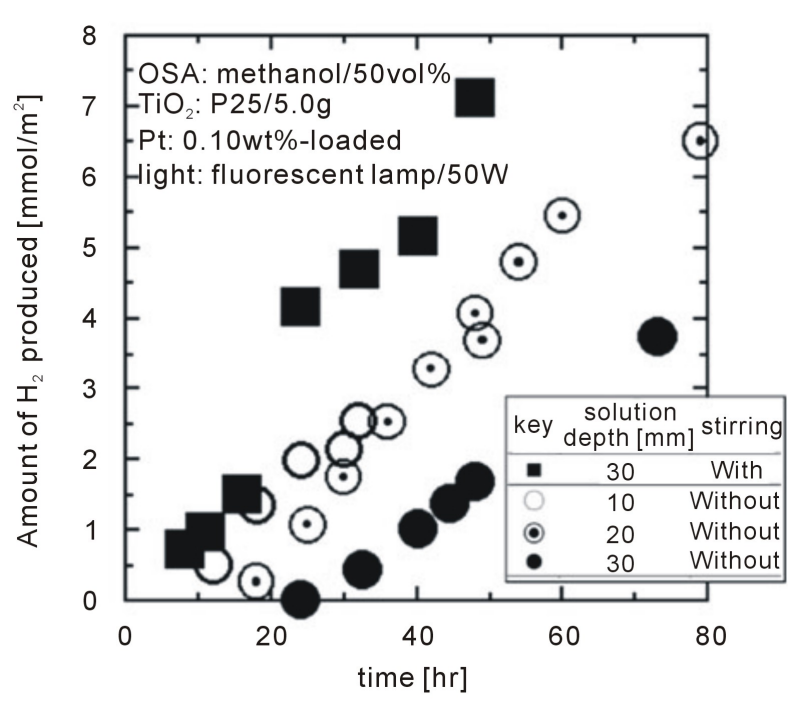

Figure 3. Effect of solution depth on photocatalytic water splitting together with indicating significance of agitation.

\subsection{Water Splitting Rates under Different Heating Conditions}

Figure 4 shows $\mathrm{H}_{2}$ producing rates with and without heating operations from preheated water at $313 \mathrm{~K}$ and 353 $\mathrm{K}$ under two solution depths of $20 \mathrm{~mm}$ and $40 \mathrm{~mm}$. In order to eliminate another potential heating source in the form of radiation from the light, black lights with lower light intensities in the visible and infrared regions were utilized instead of the fluorescent lamp used for the experiments shown in Figure 3. Furthermore, since a superior $\mathrm{TiO}_{2}$ powder named P90 for water splitting was developed by Nippon Aerosil Co., Ltd., P25 utilized until that point was replaced by P90 [9]. The amount of P90 fed to the reactor for each experiment was dared to reduce to $2.0 \mathrm{~g}$ for slower $\mathrm{H}_{2}$ producing rates in order to evaluate the efficiency of Rayleigh convection with ease.

It is clear after examining data shown in Figure 4 that heating operations (i.e. Rayleigh convection) contributed to enhance photocatalytic water splitting rates, which were slightly more than double the corresponding rates in the absence of heating. Rate enhancements obtained at $313 \mathrm{~K}$ were relatively small due to low Rayleigh convective flow rates, which were precisely described below.

\subsection{Efficiency Confirmation of Rayleigh Convections}

From Figure 3 and Figure 4, it can be seen that the shallower solutions indicate the higher photocatalytic water splitting rates without exception. This may be attributed to longer diffusing distances for the generated $\mathrm{H}_{2}$ to the gas phase as well as greater absorption/reflection ratios of ultraviolet rays in the deeper solutions.

In order to confirm the efficiency of Rayleigh convection on the rate enhancement of photocatalytic water splitting, the highest rate thus far (i.e. 20 mm solution depth with Rayleigh convection heating at $353 \mathrm{~K}$ ) shown in Figure 4 was compared with the case under a very shallow solution of only $1.4 \mathrm{~mm}$ depth with no additional heating in Figure 5. It can be seen that photocatalytic $\mathrm{H}_{2}$ production under the $1.4 \mathrm{~mm}$ depth of solution was directly proportional to time elapsed with no dead time before detecting $\mathrm{H}_{2}$, but the rate was slightly lower than the case with Rayleigh convection, despite the deeper solution of $20 \mathrm{~mm}$. Although further precise investigations are still needed to determine optimal conditions for the highest photocatalytic water splitting rate in terms of so 


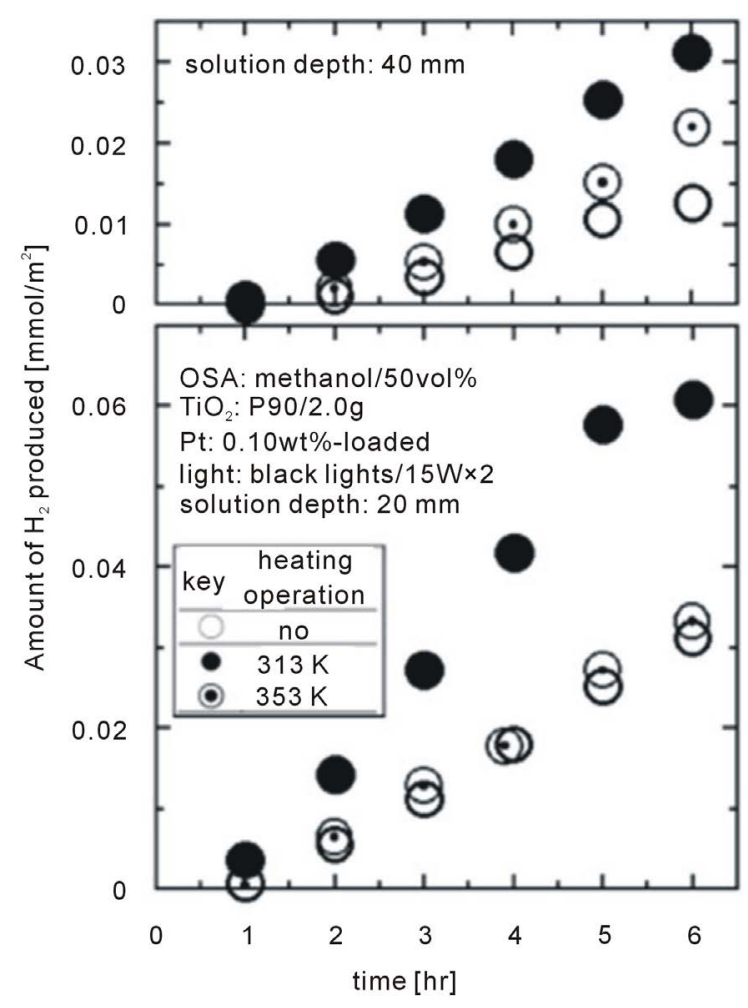

Figure 4. Effect of Rayleigh convection on photocatalytic water splitting with different depths.

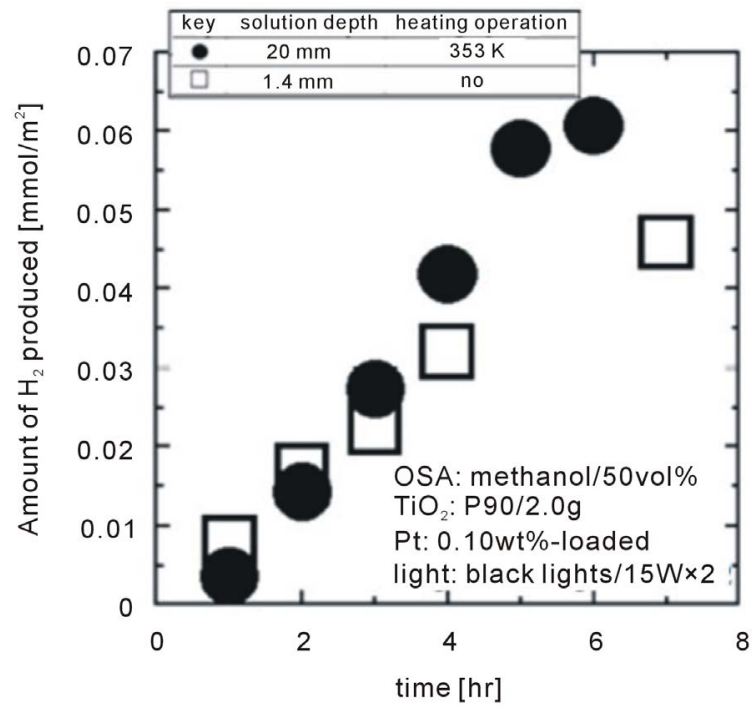

Figure 5. Significance confirmation of Rayleigh convection comparing with shallow solution case.

lution depth and the heating operation (i.e. Rayleigh convection), it can be concluded that Rayleigh convection significantly enhances photocatalytic water splitting rates.

\subsection{Efficiency Confirmation of Rayleigh Convections}

Table 2 indicates time trends of maximum and minimum Rayleigh convective flow rates in the vertical direction 
in each grid as shown in Figure 2 obtained by the conventional thermo-hydrodynamic method. It can be seen that higher Rayleigh convective flow rates are obtained at a higher heating temperature of $353 \mathrm{~K}$. Although rather low Rayleigh convective flow rates are due to such low heating temperatures, a little more than doubled the photocatalytic water splitting rates as shown in Figure 4 might result from dispersing ultrafine $\mathrm{Pt} / \mathrm{TiO}_{2}$ powder, that is to say, volumetric reactions. Therefore, Rayleigh convection was concluded to be an adequate agitating method for solid-catalyzed reactions in liquids as well as the present case.

\subsection{Strategies for Further Rate Enhancement}

Upon careful examination of experimental results, it can be seen that the slope of the line of the photocatalytic water splitting rate was decreasing after 5 hours in $20 \mathrm{~mm}$ solution depth at $353 \mathrm{~K}$ as shown in Figure 4 and Figure 5. This can be attributed to saturated Rayleigh convective flow at that time as shown in Table 2. Maintaining high Rayleigh convective flow rates without consuming any power is perhaps the most significant benefit of this technique. Future strategies for further rate enhancements are proposed in Table 3.

In the current setup, passive temperature gradients were formed by introducing preheated water to a restrictive area beneath the aluminum plate created by a grid of adiabatic Styrofoam blocks. This may eventually lead the whole system (i.e. water, OSAs, photocatalysts and facility) to the temperature of the preheated water, which would halt Rayleigh convective flow at some point in time. Therefore, simultaneous cooling along with the heating process should be needed to maintain Rayleigh convective flow. Our group proposes year-round utilization of naturally available subterranean water at around $293 \mathrm{~K}$ and relatively high pressure as a potentially viable coolant.

The Novec series manufactured by Sumitomo 3M Limited are quite unique fluids with outstanding properties of water insolubility, high relative densities (above 1) and low boiling temperatures. For example, Novec ${ }^{\mathrm{TM}} 7000$ has a water solubility level of only $50 \mathrm{ppm}$, a relative density of 1.41 and a boiling point of $307 \mathrm{~K}$. If No$\mathrm{vec}^{\mathrm{TM}} 7000$ was added to the mixtures of water, OSAs and photocatalysts, it would settle down and boil on the heated area of the aluminum plate, inducing a forced convective flow. Higher photocatalytic $\mathrm{H}_{2}$ producing rates were qualitatively confirmed by addling a slight amount of Novec $^{\mathrm{TM}} 7000$.

\section{Conclusion}

The effectiveness of Rayleigh convective flow can be experimentally confirmed for enhancing photocatalytic water splitting rates, resulting in approximately doubled rates. Since the induced Rayleigh convective flow is rather low, such rate enhancements might result from dispersed ultrafine $\mathrm{Pt} / \mathrm{TiO}_{2}$ particles throughout the whole medium, (i.e. volumetric reactions). Taking all outcomes obtained and future strategies into consideration, this thermal induced flow (i.e. Rayleigh and forced convective flow) can be concluded to have a large margin for growth in the frames of multi-phases reactions such as solid-catalyzed reactions in liquids.

Table 2. Time trends of vertical Rayleigh convective flow ranges with different heating conditions.

\begin{tabular}{ccccc}
\hline \multirow{2}{*}{$\begin{array}{c}\text { Time } \\
\text { elapsed } \\
{[\mathrm{hr}]}\end{array}$} & \multicolumn{3}{c}{ Heating temperature $[\mathrm{K}]$} \\
\cline { 2 - 5 } & $\begin{array}{c}\text { Minima } \\
{[\mathrm{nm} / \mathrm{s}]}\end{array}$ & $\begin{array}{c}\text { Maxima } \\
{[\mathrm{nm} / \mathrm{s}]}\end{array}$ & $\begin{array}{c}\text { Minima } \\
{[\mathrm{nm} / \mathrm{s}]}\end{array}$ & $\begin{array}{c}\text { Maxima } \\
{[\mathrm{nm} / \mathrm{s}]}\end{array}$ \\
\hline 1 & -0.897 & 0.867 & -3.42 & 3.24 \\
2 & -0.930 & 0.843 & -3.31 & 3.11 \\
3 & -0.949 & 0.845 & -3.31 & 3.12 \\
4 & -0.958 & 0.846 & -3.31 & 3.12 \\
5 & -0.955 & 0.846 & -3.30 & 3.12 \\
\hline
\end{tabular}

Table 3. Future strategies for enhancing photocatalytic water splitting rate.

\begin{tabular}{ccc}
\hline Present cases & Strategies & Sources or chemicals \\
\hline Adiabatic (Styrofoams) & Cooling & Underground water etc. \\
Rayleigh convection & Enforced convection & Novec series etc. \\
\hline
\end{tabular}




\section{Acknowledgements}

The authors would like to thank Nippon Aerosil Co., Ltd. for their generous supply of P25 and P90 TiO 2 powders. This work is supported by "General Sekiyu R\&D Encouragement Assistance Foundation” and “TanikawaFund Promotion of Thermal Technology”. This financial support is gratefully acknowledged. Finally, Seiichi Deguchi would like to express deep gratitude to the late Mr. Tatsumi Imura for his continual kindhearted encouragement and helpful suggestions.

\section{References}

[1] Deguchi, S., Katsuki, R., Sugiura, Y., Takeichi, T., Shibata, N. and Isu, N. (2011) Induction by Visible Light of Photocatalytic Water Decontamination by Use of Powders of Nonlinear Optic Material and Visible-Light Phosphor to Generate Dispersed Ultraviolet Light. Kagaku Kogaku Ronbunshu, 37, 38-41.

http://dx.doi.org/10.1252/kakoronbunshu.37.38

[2] Deguchi, S., Sugiura, Y., Shibata, N., Katsuki, R., Takeichi, T. and Isu, N. (2011) Photocatalytic Water Decontamination with Dispersed Light Source of Ultraviolet Electroluminescence Powder. Kagaku Kogaku Ronbunshu, 37, 42-45. http://dx.doi.org/10.1252/kakoronbunshu.37.42

[3] Hong, J., Sun, S., Yang, S.G. and Liu, Y.Z. (2006) Photocatalytic Degradation of Methylene Blue in $\mathrm{TiO}_{2}$ Aqueous Suspensions Using Microwave Powered Electrodeless Discharge Lamps. Journal of Hazardous Materials, 133, 162166. http://dx.doi.org/10.1016/j.jhazmat.2005.10.004

[4] Horikoshi, S., Kajitani, M., Sato, S. and Serpone, N. (2007) A Novel Environmental Risk-Free Microwave Discharge Electrodeless Lamp (MDEL) in Advanced Oxidation Processes, Degradation of the 2,4-D Herbicide. Journal of Photochemistry and Photobiology A: Chemistry, 189, 355-363. http://dx.doi.org/10.1016/j.jphotochem.2007.02.027

[5] Mueller, P., Loupy, A. and Klan, P. (2005) The Electrodeless Discharge Lamp: A Prospective Tool for Photochemistry. Journal of Photochemistry and Photobiology A: Chemistry, 172, 146-150. http://dx.doi.org/10.1016/j.jphotochem.2004.12.003

[6] Deguchi, S., Shibata, N., Takeichi, T., Furukawa, Y. and Isu, N. (2010) Photocatalytic Hydrogen Production from Aqueous Solution of Various Oxidizing Sacrifice Agents. Journal of the Japan Petroleum Institute, 53, 95-100.

[7] Deguchi, S., Takeichi, T., Shimasaki, S., Ogawa, M. and Isu, N. (2011) Photocatalytic Hydrogen Production from Water with Nonfood Hydrocarbons as Oxidizing Sacrifice Agents. AIChE Journal, 57, 2237-2243. http://dx.doi.org/10.1002/aic.12414

[8] Matsuoka, M., Kitano, M., Takeuchi, M., Tsujimaru, K., Anpo, M. and Thomas, J.M. (2007) Photocatalysis for New Energy Production: Recent Advances in Photocatalytic Water Splitting Reactions for Hydrogen Production. Catalysis Today, 122, 51-61. http://dx.doi.org/10.1016/j.cattod.2007.01.042

[9] Deguchi, S., Kobayashi, N. and Kubota, M. (2008) Environmental Purifying Materials, Equipment and Method. PATENT in Japan, No. 2008-194622.

[10] Hijikata, T. (2002) Research and Development of International Clean Energy Network Using Hydrogen Energy (WENET). International Journal of Hydrogen Energy, 27, 115-129. http://dx.doi.org/10.1016/S0360-3199(01)00089-1

[11] Sato, S. and White, J.M. (1980) Photoassisted Water-Gas Shift Reaction over Platinized Titanium Dioxide Catalysts. Journal of the American Chemical Society, 102, 7206-7210. http://dx.doi.org/10.1021/ja00544a006

[12] Chen, B., Mikami, F. and Nishikawa, N. (1999) Numerical Simulation of Natural Convection in Particle Suspensions. Transactions of the Japan Society of Mechanical Engineering B, 65, 1200-1207. http://dx.doi.org/10.1299/kikaib.65.1200

[13] Nakano, A., Shigechi, T. and Momoki, S. (2004) Numerical Analysis of Natural Convection from a Heated Plate Facing Downwards. Transactions of the Japan Society of Mechanical Engineering B, 70, 1797-1803. http://dx.doi.org/10.1299/kikaib.70.1797

[14] Matsumoto, Y. and Kameda, M. (1993) Propagation of Shock Waves in Dilute Bubbly Liquids. Transactions of the Japan Society of Mechanical Engineering B, 69, 2386-2393. http://dx.doi.org/10.1299/kikaib.59.2386

[15] Hernandez, R. (1995) Influence of the Heating Rate on Supercritical Rayleigh-Benard Convection. International Journal of Heat and Mass Transfer, 38, 3035-3051. http://dx.doi.org/10.1016/0017-9310(95)00023-3 